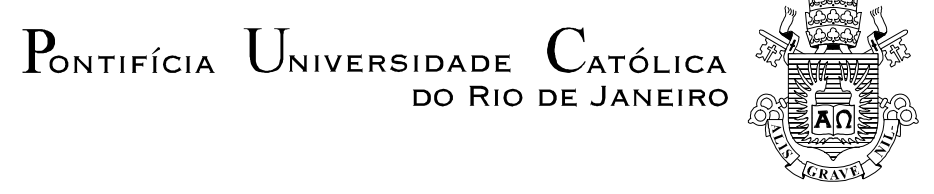

Guilherme de Araújo Szundy

Modelagem e Implementação de Aplicações Hipermídia Governadas por Ontologias para a Web Semântica

Dissertação de Mestrado

Dissertação apresentada como requisito parcial para obtenção do título de Mestre pelo Programa de PósGraduação em Informática da PUC-Rio.

Orientador: Daniel Schwabe

Rio de Janeiro, novembro de 2004 


$$
\text { Pontifícia } \text { Universidade }_{\text {Do Rio de Janeiro }}
$$

Guilherme de Araújo Szundy

\title{
Modelagem e Implementação de Aplicações Hipermídia Governadas por Ontologias para a Web Semântica
}

\begin{abstract}
Dissertação apresentada como requisito parcial para obtenção do título de Mestre pelo Programa de PósGraduação em Informática da PUC-Rio. Aprovada pela Comissão Examinadora abaixo assinada.
\end{abstract}

Daniel Schwabe

Orientador

PUC-Rio

Fernanda Lima

Universidade Católica de Brasília

Marco Antônio Casanova

PUC-Rio

Simone Diniz Junqueira Barbosa

PUC-Rio

Karin Koogan Breitman

PUC-Rio

Prof. José Eugenio Leal

Coordenador(a) Setorial do Centro Técnico Científico - PUC-Rio

Rio de Janeiro, 10 de novembro de 2004 
Todos os direitos reservados. É proibida a reprodução total ou parcial do trabalho sem autorização da universidade, do autor e do orientador.

\section{Guilherme de Araújo Szundy}

Graduou-se em Engenharia de Computação na PUC-Rio em 1999. Atuou como Analista de Sistemas e Programador no desenvolvimento de soluções web para organizações não governamentais, instituições educacionais, bancos, grupos de mídia, governos e algumas das maiores empresas privadas do país. Possui interesse acadêmico e profissional nas áreas de Hipertexto e Multimídia, Engenharia de Software e Interação Humano-Computador.

Ficha Catalográfica

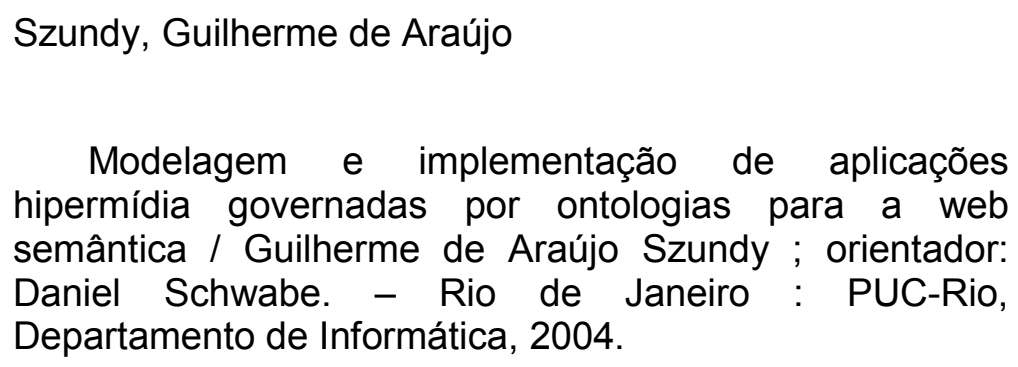

Modelagem e implementação de aplicações hipermídia governadas por ontologias para a web semântica / Guilherme de Araújo Szundy ; orientador: Daniel Schwabe. - Rio de Janeiro : PUC-Rio, Departamento de Informática, 2004.

154 f. : il. ; $30 \mathrm{~cm}$

Dissertação (mestrado) - Pontifícia Universidade Católica do Rio de Janeiro, Departamento de Informática.

Inclui referências bibliográficas.

1. Informática - Teses. 2. Aplicações hipermídia. 3. Aplicações Web. 4. OOHDM. 5. SHDM. 6. Web semântica. I. Schwabe, Daniel. II. Pontifícia Universidade Católica do Rio de Janeiro. Departamento de Informática. III. Título. 
Este trabalho é dedicado a minha família, pelo carinho e apoio incondicional, e ao meu orientador, pelo exemplo e inspiração. 


\section{Agradecimentos}

À PUC-Rio e ao departamento de informática pela oportunidade concedida.

Ao meu orientador, Professor Daniel Schwabe, pelo incentivo e confiança.

Aos membros da comissão examinadora pela paciência e atenção.

Aos colegas de estudo e pesquisa João, Sabrina e Adriana pelo apoio e pelas ricas trocas de idéias.

A todos os professores, funcionários e alunos do Departamento de Informática pela convivência agradável de todos os dias.

A Deborah, Emanuelle e Ruth pelo socorro nos trâmites burocráticos.

Aos meus amigos e familiares por tornarem tudo mais divertido e pelo apoio nos momentos difíceis.

A minha querida irmã Renata e meu cunhado Plínio pela amizade sempre presente.

Ao meu sobrinho Felipe pela alegria e simplicidade de ser criança.

Aos meus amados pais Alexandre e Célia por tudo. 


\section{Resumo}

Szundy, Guilherme de Araújo; Schwabe, Daniel. Modelagem e Implementação de Aplicações Hipermídia Governadas por Ontologias para a Web Semântica. Rio de Janeiro, 2004. 154p. Dissertação de Mestrado - Departamento de Informática, Pontifícia Universidade Católica do Rio de Janeiro.

Essa dissertação propõe um modelo para o desenvolvimento de aplicações hipermídia na Web Semântica estendendo o método SHDM (Semantic Hypermedia Design Method), e apresenta uma arquitetura de implementação deste modelo. $\mathrm{Na}$ extensão proposta, as aplicações são visões navegacionais especificadas sobre modelos conceituais definidos por quaisquer ontologias da Web Semântica. As classes navegacionais são caracterizadas por um padrão para recuperação de dados da ontologia conceitual a partir de um recurso específico, podendo incorporar regras para filtragem de instâncias. Estruturas de acesso passam a se distinguir quanto à origem de seus dados, podendo ser arbitrárias, derivadas de consultas, derivadas de contextos ou facetadas. Elos especializam um relacionamento com base nos tipos de sua origem e destino, e podem ser definidos a partir de uma composição de relacionamentos conceituais. A especificação do modelo navegacional é dada através de um vocabulário definido como uma ontologia, tornando-a independente do ambiente de inferência e consulta empregado em qualquer implementação do modelo. Os modelos propostos são utilizados como dados para a geração de aplicações, com suporte na arquitetura de implementação definida e implementada.

\section{Palavras-chave}

Aplicações Hipermídia; Aplicações Web; OOHDM; SHDM; Web; Web Semântica; WWW 


\section{Abstract}

Szundy, Guilherme de Araújo; Schwabe, Daniel (Advisor). Modeling and Implementation of Ontology Driven Hypermedia Applications for the Semantic Web. Rio de Janeiro, 2004. 154p. MSc. Dissertation Departamento de Informática, Pontifícia Universidade Católica do Rio de Janeiro.

This dissertation proposes a development model for hypermedia applications on the Semantic Web extending the Semantic Hypermedia Design Method (SHDM), and presents an implementation architecture for this model. In the proposed extension applications are seen as navigational views over conceptual models defined by any Semantic Web ontology. Navigational classes are characterized by a retrieval pattern of conceptual ontology data from a specific resource, and can also incorporate rules for instances filtering. Access structures are now distinguished by their data source, and are categorized as arbitrary, query based, context based or faceted. Links specialize conceptual relationships based on the data types of its origin and destination, and can also map relationship compositions. A vocabulary defined as an ontology is used for the specification of the navigational model, making it independent from the query and inference environment used by any implementation of the model. The implementation architecture specified and implemented generates applications directly from the data contained in the proposed models.

\section{Keywords}

Hypermedia Applications; Web Applications; OOHDM; SHDM; Web; Semantic Web; WWW 


\section{Sumário}

1 Introdução 19

1.1 A Web Semântica 19

1.2 Explorando dados na Web Semântica 22

1.3 Organização da dissertação 24

2 O método SHDM 25

2.1 Modelo conceitual $\quad 29$

2.2 Modelo navegacional 34

2.2.1 Classes navegacionais 35

$\begin{array}{ll}2.2 .2 \text { Classes em contexto } & 37\end{array}$

$\begin{array}{ll}2.2 .3 \text { Elos } & 38\end{array}$

2.2.4 Contextos navegacionais 40

2.2.5 Estruturas de acesso 42

2.2.6 Navegação facetada 43

2.2.7 Landmarks 46

2.3 Operações 46

3 Especificação do mapeamento navegacional SHDM 48

3.1 Mapeamento de classes navegacionais 51

3.1.1 Mapeamento de instâncias $\quad 51$

3.1.2 Definição de atributos $\quad 54$

3.1.2.1. Atributos simples $\quad 54$

3.1.2.2. Listas $\quad 57$

3.1.2.3. Atributos como âncoras 65

3.1.2.4. Atributos como índices 67

$\begin{array}{ll}3.1 .3 \text { Generalização } & 68\end{array}$

$\begin{array}{ll}3.2 \text { Mapeamento de elos } & 69\end{array}$

$\begin{array}{ll}3.3 \text { Classes em contexto } & 70\end{array}$

$\begin{array}{ll}3.4 \text { Contextos navegacionais } & 71\end{array}$ 
$\begin{array}{ll}3.5 \text { Estruturas de acesso } & 78\end{array}$

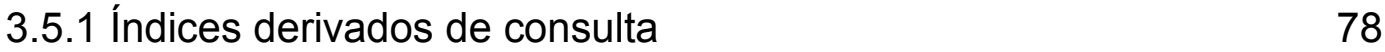

3.5.2 Índices derivados de contexto 80

$\begin{array}{ll}3.5 .3 \text { Índices arbitrários } & 81\end{array}$

3.5.4 Índices facetados $\quad 83$

3.6 Facetas e grupos de facetas $\quad 84$

3.7 Landmarks $\quad 89$

$\begin{array}{ll}3.8 \text { Operações } & 89\end{array}$

4 Exemplo 91

4.1 Modelo conceitual $\quad 91$

4.2 Mapeamento navegacional 92

5 Arquitetura de implementação 103

5.1 Visão geral 103

5.2 Implementação 104

5.2.1 O modelo de visualizações 108

5.2.2 O módulo de execução 109

5.2.3 Leitura e mapeamento 109

5.2.4 O tratamento de uma requisição 109

5.2.5 Funcionalidades implementadas 111

6 Conclusão 112

6.1 Trabalhos relacionados 112

6.2 Contribuições 114

$\begin{array}{ll}\text { 6.3 Trabalhos futuros } & 115\end{array}$

7 Referências bibliográficas 118

8 Apêndice I - Vocabulário SHDM 121

8.1 Classes 121

$\begin{array}{ll}8.2 \text { Propriedades } & 123\end{array}$

9 Apêndice II - Notação N3 136 
10 Apêndice III - Ontologia conceitual da aplicação exemplo

11 Apêndice IV - Especificação do mapeamento navegacional da aplicação exemplo 


\section{Siglas}

DAML

HTML

OIL

OOHDM

OWL

RDF

RDFS

RDQL

$\mathrm{RQL}$

SHDM

URI

URL

W3C

Web

WWW

$\mathrm{XML}$

XML Schema

JSP

MVC
DARPA Agent Markup Language

HyperText Markup Language

Ontology Inference Layer

Object Oriented Hypermedia Design Method

Web Ontology Language

Resource Description Framework

Resource Description Framework Schema

RDF Data Query Language

RDF Query Language

Semantic Hypermedia Design Method

Universal Resource Identifier

Uniform Resource Locator

World Wide Web Consortium

World Wide Web

World Wide Web

eXtensible Markup Language

eXtensible Markup Schema Language

Java Server Pages

Model-View-Controler 


\section{Lista de tabelas}

Tabela 1 - Artefatos do método SHDM 28

Tabela 2 - Prefixos dos namespaces utilizados na dissertação 30

Tabela 3 - Exemplo de tabela de mapeamento de classe navegacional 37

Tabela 4 - Lista com dados das músicas de um CD 58

Tabela 5 - Lista de CDs sem ordenação 63

Tabela 6 - Lista de CDs ordenados por título 63

Tabela 7 - Lista de CDs ordenados por ano e título 63

Tabela 8 - Alternativas para declaração da etiqueta de um atributo âncora definido sobre um elo $\quad 66$

Tabela 9 - Classes do vocabulário SHDM 123 


\section{Lista de figuras}

Figura 1 - Exemplo de um grafo RDF

Figura 2 - Fluxo típico de desenvolvimento de uma aplicação pelo método SHDM

Figura 3 - Exemplo de representação de um esquema conceitual como um diagrama de classes no estilo UML

Figura 4 - Exemplo da aplicação da máscara de uma classe navegacional em um grafo RDF 36

Figura 5 - Exemplo de representação gráfica da classe navegacional Book

Figura 6 - Exemplo de mapeamento de um caminho de propriedades como um elo

Figura 7 - Exemplo do mapeamento de elos especializando as relações representadas por propriedades conceituais 39

Figura 8 - Exemplo de navegação em busca de uma obra de arte $\quad 43$

Figura 9 - Exemplo do particionamento do conjunto de obras de arte

Figura 10 - Exemplo de organização hierárquica dos valores de uma faceta de obras de artes pelo país de origem

Figura 11 - Exemplo de um grafo RDF de instâncias conceituais 49

Figura 12 - Exemplo de um grafo RDF de instâncias navegacionais $\quad 50$

Figura 13 - Exemplo de mapeamento de um atributo como uma lista 58 Figura 14 - Grafo com os dados de instâncias conceituais das faixas de um CD

Figura 15 - Árvore correspondendo ao mapeamento de um atributo como uma lista

Figura 16 - Identificação dos dados de uma lista em um grafo de instâncias conceituais

Figura 17 - Grafo de instâncias navegacionais resultante do mapeamento de uma lista

Figura 18 - Esquema conceitual para o website de um departamento acadêmico 
Figura 19 - Esquema de classes navegacionais para o website do departamento acadêmico

Figura 20 - Diagrama de contextos para o website do departamento acadêmico

Figura 21 - Cartão de contexto de alunos em ordem alfabética, ctxAlunosAlfa

Figura 22 - Cartão de contexto de pessoa por publicação, ctxPessoaPorPublicacao

Figura 23 - Cartão da estrutura de acesso idxAreasDePesquisa 98

Figura 24 - Cartão da estrutura de acesso idxProfessores 100

Figura 25 - Arquitetura para execução de aplicações SHDM 103

Figura 26 - Fluxo de processamento de uma requisição 104

Figura 27 - Arquitetura JSP Modelo 2

Figura 28 - Arquitetura de módulos do sistema 106

Figura 29 - Diagrama de pacotes do sistema 107

Figura 30 - Diagrama de classes do pacote shdm.data 108

Figura 31 - Diagrama de seqüência do processamento de uma requisição 


\section{Lista de quadros}

Quadro 1 - Exemplo de declaração de dados em RDF/XML 20

Quadro 2 - Exemplo de declaração de uma sentença RDF 29

Quadro 3 - Exemplo de declaração de um conjunto de sentenças RDF 30

Quadro 4 - Exemplo de declaração de classes em RDFS 30

Quadro 5 - Exemplo de declaração de mapeamento de classe

navegacional $\quad 50$

Quadro 6 - Exemplo de declaração de mapeamento de classe

navegacional com regra de filtragem de instâncias 52

Quadro 7 - Exemplo de declaração de mapeamento de atributo simples 54

Quadro 8 - Exemplo de declaração do mapeamento de classe navegacional com mapeamento automático de atributos simples $\quad 56$

Quadro 9 - Exemplo de declaração de mapeamento de uma classe navegacional com filtro de exclusão para mapeamento automático de atributos simples (no exemplo, ex:categoria) 56

Quadro 10 - Exemplo de declaração de atributo de uma classe navegacional como uma lista

Quadro 11 - Exemplo de declaração de atributo de uma classe navegacional como uma lista com ordenação

Quadro 12 - Exemplo de declaração de atributo de classe navegacional como âncora a partir de um elo

Quadro 13 - Exemplo de declaração de atributo de classe navegacional como âncora

Quadro 14 - Exemplo de declaração de mapeamento de índice como atributo de uma classe navegacional

Quadro 15 - Exemplo da declaração de mapeamento de classe navegacional com especialização

68

Quadro 16 - Exemplo de declaração de mapeamento de elo 69

Quadro 17 - Exemplo de declaração de mapeamento inverso de elo 69

Quadro 18 - Exemplo de declaração de classe em contexto 70 
Quadro 19 - Exemplo de declaração de um contexto com uma regra de seleção sem condição adicional

Quadro 20 - Exemplo de declaração de um contexto com duas regras de seleção

Quadro 21 - Exemplo de declaração de um contexto parametrizado $\quad 73$

Quadro 22 - Exemplo de declaração de um contexto facetado

Quadro 23 - Exemplo de declaração de um contexto com navegação circular

Quadro 24 - Exemplo de declaração de um contexto com navegação seqüencial e por índice $\quad 75$

Quadro 25 - Exemplo de declaração de interseção de contextos

Quadro 26 - Exemplo de declaração de um contexto com critério de ordenação padrão

Quadro 27 - Exemplo de declaração de um índice derivado de consulta com dois atributos

Quadro 28 - Exemplo de declaração de um índice derivado de consulta parametrizado

Quadro 29 - Exemplo de declaração de um índice derivado de contexto 80

Quadro 30 - Exemplo de declaração de um índice arbitrário 82

Quadro 31 - Exemplo de declaração de um índice facetado 83

Quadro 32 - Exemplo de declaração de um grupo de facetas 84

Quadro 33 - Exemplo de declaração de combinações inválidas de valores de facetas

Quadro 34 - Exemplo de declaração de uma faceta sobre um atributo de uma classe navegacional

Quadro 35 - Exemplo de declaração de uma faceta sobre um elo de uma classe navegacional

Quadro 36 - Exemplo de declaração de uma faceta com a especificação de uma hierarquia de valores

Quadro 37 - Exemplo de declaração de uma faceta em função de uma hierarquia de classes

Quadro 38 - Exemplo de declaração de um landmark

Quadro 39 - Exemplo de declaração de assinatura de uma operação 
Quadro 40 - Declaração da propriedade conceitual ex:nome

Quadro 41 - Declaração do mapeamento da classe navegacional Publicacao

Quadro 42 - Declaração do mapeamento do atributo titulacao da classe navegacional Professor

Quadro 43 - Declaração do contexto de alunos em ordem alfabética, ctxAlunosAlfa

Quadro 44 - Declaração do contexto de pessoa por publicação, ctxPessoaPorPublicacao

Quadro 45 - Declaração da estrutura de acesso idxAreasDePesquisa 98

Quadro 46 - Declaração da estrutura de acesso idxProfessores $\quad 100$

Quadro 47 - Exemplo de uma tripla RDF expressa em N3 135

Quadro 48 - Exemplo de uso de prefixos de namespace em N3 136

Quadro 49 - Exemplo de representação de uma sentença RDF na sintaxe RDF/XML

Quadro 50 - Exemplo de repetição de um sujeito em diversas sentenças

Quadro 51 - Exemplo do uso de ponto-e-vírgula para evitar a repetição do sujeito em diversas sentenças

Quadro 52 - Exemplo de repetição de uma mesma propriedade na descrição de um recurso

Quadro 53 - Exemplo do uso da vírgula para evitar e repetição de uma mesma propriedade na descrição de um recurso

Quadro 54 - Exemplo da descrição de um recurso em RDF/XML

Quadro 55 - Exemplo de declaração de um contexto em RDF/XML 138

Quadro 56 - Exemplo de declaração de um contexto em N3

Quadro 57 - Exemplo de declaração do tipo de um recurso, em N3, utilizando a propriedade $r d f: t y p e$

Quadro 58 - Exemplo de declaração do tipo de um recurso, em N3, utilizando a abreviação da propriedade rdf:type

Quadro 59 - Exemplo de declaração do tipo de um recurso em RDF/XML 
Quadro 60 - Exemplo de declaração do tipo de um recurso, em RDF/XML, em formato compacto 